\title{
Superiority of Two-Dimensional Echocardiography in the Diagnosis of Congenitally Bicuspid Aortic Valve
}

\author{
Veena Raizada, M.D., Ron Roth, M.D., \\ Jonathan Abrams, M.D., and Ken Schroeder
}

\section{Summary}

This study compares the role of $\mathbf{M}$-mode and two-dimensional echocardiography in the evaluation of the nonstenotic, congenitally bicuspid aortic valve. Ten asymptomatic healthy subjects (age 2035 years) with a systolic ejection click and short systolic murmur were evaluated. M-mode echocardiograms were entirely normal. The aortic root, diastolic closure line, eccentricity index of the aortic valve, and left ventricular size and function were normal in every subject. The two-dimensional parasternal long axis view of the aortic root was also normal in all patients with bicuspid aortic valves; there were no abnormalities of systolic excursion of the aortic cusps and doming of the aortic valve was absent. Images obtained in the two-dimensional parasternal short axis view of the aortic root at the level of the aortic valve revealed only two cusps, anterior and posterior, in all patients. The classic closure pattern of the normal tricuspid aortic valve forming a " $\mathrm{Y}$ " was absent in every subject. Instead, a horizontal closure line along the "X" axis was demonstrated in all patients; changing the angulation of the transducer in various positions did not alter this pattern. All subjects had increased reflectance of echoes along the line of diastolic closure, probably resulting from redundancy of the aortic cusp tissue. We conclude that because of the inability of the M-mode echocardiographic technique to accurately diagnose this condition in the study group, two-dimensional echocardiography should be the technique of choice in the evaluation of patients with a suspected bicuspid aortic valve. Recognition of this condition is important in view of its susceptibility to subacute bacterial endocarditis, aortic stenosis, and regurgitation.

\section{Additional Indexing Words:}

Bicuspid aortic valve

From the Division of Cardiology, Department of Medicine, University of New Mexico School of Medicine, Albuquerque, New Mexico 87131, U.S.A.

Address for reprint: Veena Raizada, M.D., Division of Cardiology, Department of Medicine, University of New Mexico School of Medicine, Albuquerque, New Mexico 87131, U.S.A.

Received for publication July 22, 1981. 
R ICUSPID aortic valve is the most common congenital abnormality of the 3 heart, occurring in approximately $1-2 \%$ of routine autopsies. ${ }^{11,2)}$ The potential for infective endocarditis, ${ }^{3), 4}$ or progression to aortic stenosis and regurgitation ${ }^{51-9)}$ make it desirable to identify this abnormality by a reliable noninvasive method. The diagnosis of a bicuspid aortic valve should initiate prophylaxis against endocarditis and a follow-up program for early detection of subsequent complications. The ability of M-mode echocardiography to detect a bicuspid aortic valve has been amply documented; the criteria include an abnormal eccentricity index of diastolic closure and multilayering of echoes during diastole. ${ }^{10,11)}$ Wide variability of the eccentricity index, and a substantial overlap of this index in patients with deformed tricuspid and bicuspid aortic valves, make this parameter less sensitive and specific than desired.11),12) Fowles et al ${ }^{12}$ ) have emphasized the superiority of two-dimensional echocardiography in the diagnosis of both stenotic and non stenotic bicuspid aortic valves and associated abnormalities of the valve cusps. This study describes the two-dimensional echocardiographic findings of the non-stenotic, congenitally bicuspid aortic valve in a young adult population who were evaluated for the presence of a systolic ejection click and short systolic murmur without other manifestations of organic heart disease.

\section{Materials and Methods}

\section{Patient selection}

This series consists of 10 asymptomatic patients clinically suspected of a bicuspid aortic valve (age range 20-35 yrs) and 10 age and sex matched normal control subjects. These patients had been seen in the general medical clinic for heart murmur evaluation and were subsequently referred to the echocardiographic laboratory for confirmation of a suspected bicuspid aortic valve. An early systolic click without respiratory variation and a short systolic murmur were present in all subjects (Fig. 1). Two patients had a short aortic regurgitant murmur. Every subject demonstrated normal systolic excursion of the aortic valve cusps both on M-mode and two-dimensional echocardiograms, thus ruling out aortic stenosis by the current echocardiographic criteria. ${ }^{13)-15}$ ) None of these patients had clinical or phonocardiographic evidence to suggest involvement of the other cardiac valves. The absence of respiratory variation of the click was felt to exclude pulmonic stenosis. ${ }^{17}$ ) There was no evidence of rheumatic heart disease, mitral valve prolapse, systemic hypertension, or connective tissue disorders in any of these patients. In view of the fact that none of these patients had clinical echocardiographic evidence of hemodynamically significant aortic valve diseases we elected not to subject these pa- 

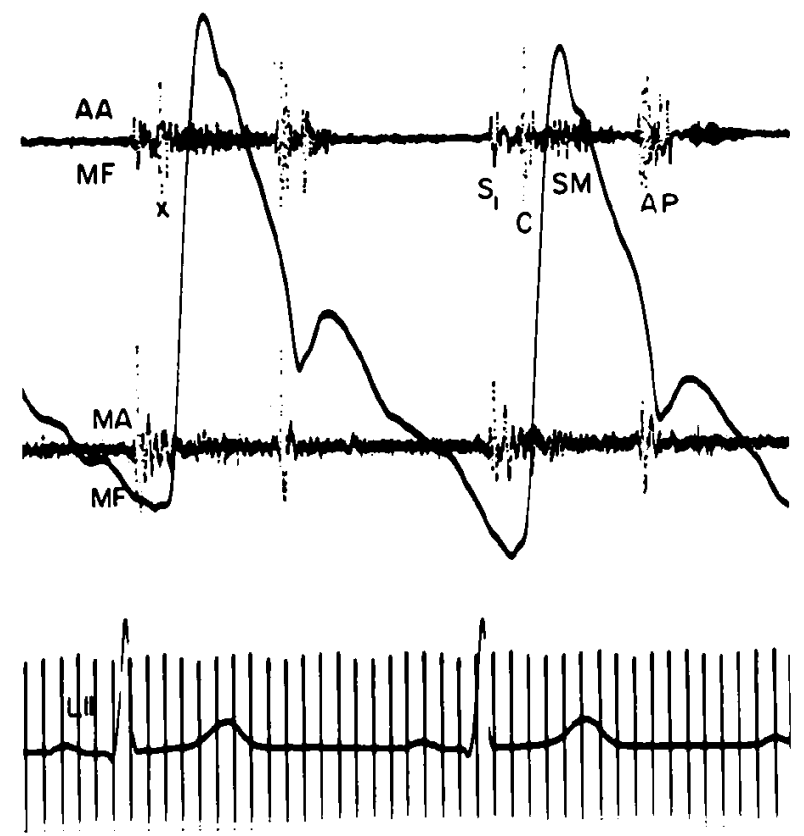

Fig. 1. Phonocardiogram showing medium frequency sound tracing recorded at the aortic and the mitral areas, with the carotid pulse and lead II of electrocardiogram. An aortic ejection click (X) preceding a short ejection murmur is shown. $A=$ aortic component of second heart sound; $A A=$ aortic area $\mathrm{C}=$ systolic click; LII = lead II of the electrocardiogram; MA=mitral area; $M F=$ medium frequency; $P=$ pulmonic component of the second heart sound; $S_{1}=$ first heart sound.

tients to a left heart catheterization and aortography.

\section{Methods}

M-mode ${ }^{13), 14)}$ and two-dimensional echocardiography ${ }^{18)}$ were performed on all patients by standard techniques. M-mode echocardiograms of the aortic root and valve were recorded on a strip chart using a $2.25 \mathrm{MHz}, 1 / 2$ inch diameter transducer focused at $5 \mathrm{~cm}$. The aortic valve was recognized by the typical box-like opening and closing movements of the aortic valve cusps within the lumen of the aortic root. ${ }^{13)}$ Cross-sectional echocardiography was performed with a commercially available mechanical sector scanner (Advance Technology Laboratories), with three rotating $3.0 \mathrm{MHz}$ elements. In each subject, two-dimensional echocardiograms were recorded in a plane parallel to the long axis of the aorta as well as in the short axis of the aorta at the level of the aortic valve in the supine and left lateral decubitus. The images were recorded and played back on a Sanyo video tape recording system. The illustrations presented in this report were obtained from the photographs of the still frames of the cross-sectional images and therefore are not entirely 
representative of the quality of the echocardiographic studies obtained by real time two-dimensional technique.

\section{Results}

\section{Control subjects}

Both M-mode and two-dimensional echocardiographic parasternal long axis images of the aortic valve showed normal systolic excursion of the cusps $(15-20 \mathrm{~mm})$; diastolic closure was seen as a single line in the center of the aortic lumen; the eccentricity index was less than 1.3 in all subjects (Fig. 2a). In the short axis view of the aortic root at the level of the aortic valve, the three aortic cusps and commissures formed a " $\mathrm{Y}$ " pattern during diastole in every subject (Fig. 2b). Simultaneous demonstration of the entire lengths of the commissures between the coronary and noncoronary cusps was somewhat difficult on the still frames. The upper part of the "Y" was visualized as a shallow "V" rather than a horizontal line; the positions of the commissures were deduced from careful analysis of several frames in real time (Fig. 2c, d).

\section{Patients with bicuspid aortic valve}

The size of the aortic root, systolic excursion, and diastolic closure of the aortic valve cusps and the calculated eccentricity index were normal $(<1.3)$ on the M-mode echocardiograms in all patients (Fig. 3a). Multilayering of echoes during diastole was not seen in any subject.

In the two-dimensional echocardiographic parasternal long axis image of the aortic root, the right coronary, and the posterior non-coronary cusps opened normally during systole, and systolic doming of the cusps was absent; diastolic closure was located in the center of the aortic lumen in each subject (Fig. 3b). However, in the short axis view, only two cusps, the anterior and the posterior, were seen in each patient. The classic closure pattern of a normal tricuspid aortic valve forming a " $\mathrm{Y}$ " was absent (Fig. 3c); instead a horizontal closure line along the $\mathrm{X}$ axis representing the commissure at the junction of the upper and lower halves was demonstrated in all patients. There was increased reflectance of the echoes from the line of closure of the cusps. Changing the angulation of the transducer in various positions did not alter these patterns. The left ventricle, left atrium, mitral valve, right heart structures, and pericardium were normal in every subject.

\section{Discussion}

Despite certain limitations of M-mode and two-dimensional echocardio- 


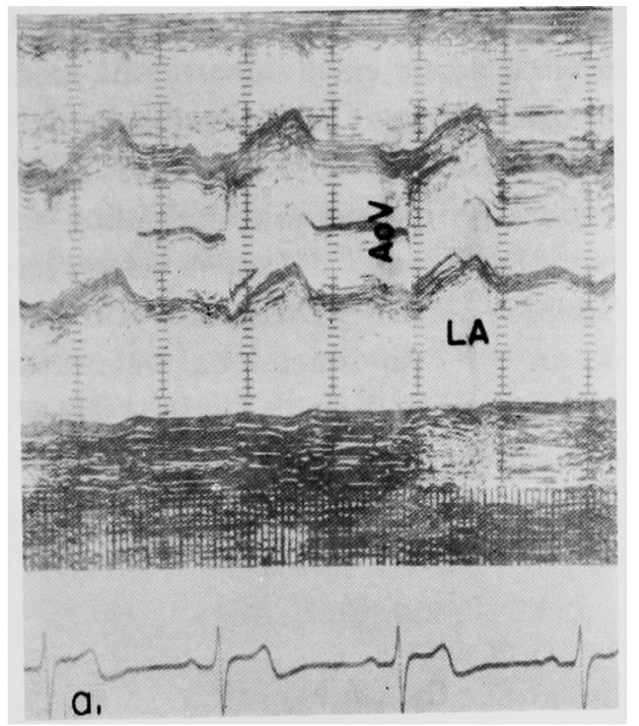

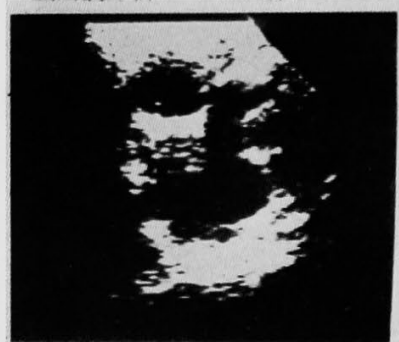

b.

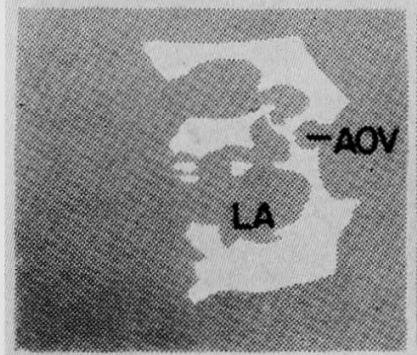

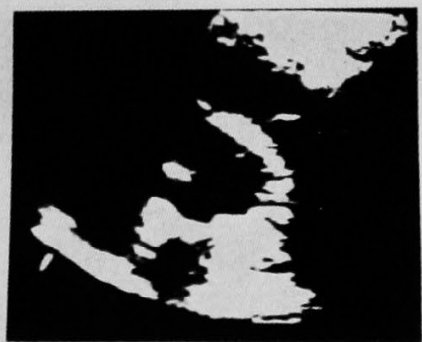

c.

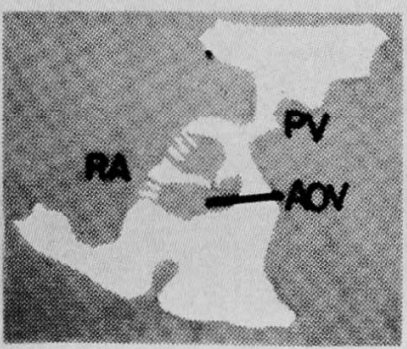

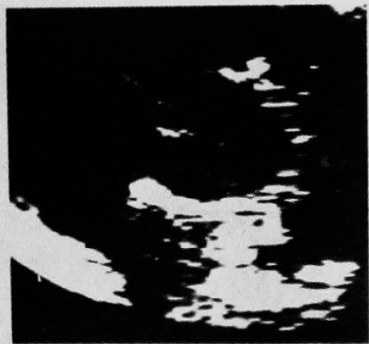

d.

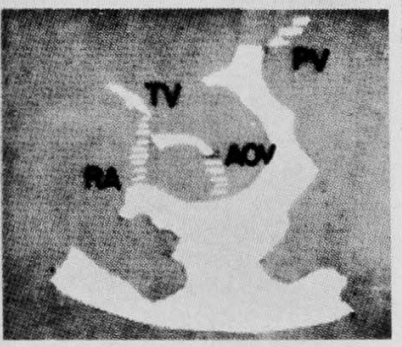

Fig. 2. Normal aortic valve (Panel a) shows M-mode echocardiogram of a normal aortic valve with mid-aortic diastolic closure of the cusps; systolic opening of the cusps in a box-like configuration is also shown. Parasternal short axis two-dimensional echocardiographic image of the aortic root at the level of the aortic valve (Panels b, c, and d). In a normal tricuspid aortic valve, the margins of the closed aortic cusps form a classic " $Y$ " pattern (Panel b, schematic diagram). Panels $c$ and $d$ demonstrate positions of the commissures between the three cusps of the aortic valve (see text for explanation). $A O V=$ aortic valve; $\mathrm{LA}=$ left atrium; $\mathrm{RA}=$ right atrium; $\mathrm{PV}=$ pulmonic valve; $T V=$ tricuspid valve. 
graphy, these techniques have proved to be clinically useful in the evaluation of congenital and acquired aortic valve disease. M-mode echocardiographic criteria for diagnosis of a bicuspid aortic valve include eccentric diastolic closure of the cusps, with an eccentricity index 1.3 or greater, ${ }^{101,11)}$ and multilayering of valve echoes during diastole, providing there is no associated high membranous ventricular septal defect. ${ }^{11}$ However, these criteria have not proved to be as useful as initially predicted; false positive and false negative diagnoses do occur. ${ }^{12)}$ Two-dimensional echocardiography allows examination of the cardiac structures in multiple cross-sectional planes, ${ }^{18}$ ) and the advantage of increased spatial orientation is especially helpful in assessing complex anatomical structures such as the bicuspid aortic valve.

In our patients with the clinical diagnosis of a possible bicuspid aortic valve, the $\mathrm{M}$-mode echocardiogram was entirely normal in every subject. There was no abnormality of the aortic cusps in the two-dimensional parasternal long axis view of the aorta. However, in the short axis view of the aortic

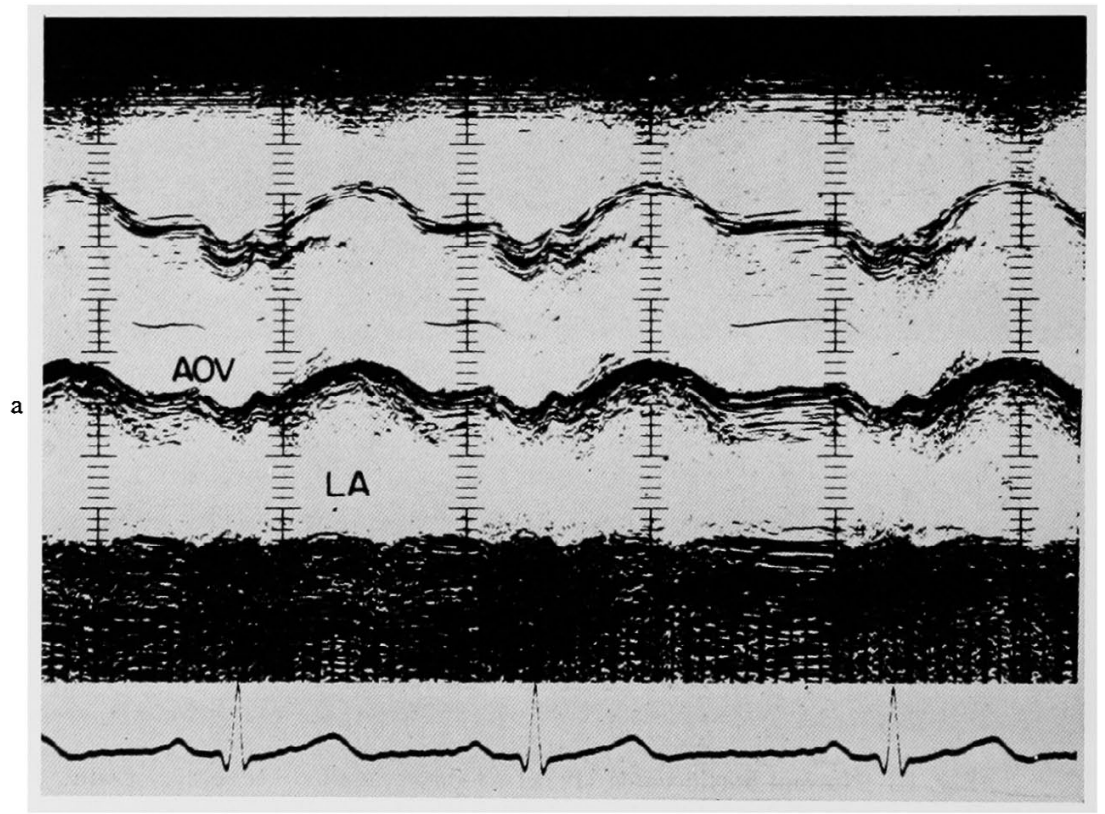

Fig. 3. Bicuspid aortic valve: M-mode echocardiogram (Panel a) showing a mid-aortic, central diastolic closure of the cusps, a normal eccentricity index, and absence of multiple dense echoes during diastole. Parasternal long and short axis two-dimensional echocardiographic images of the aorlic root at the level of the aortic valve are shown in Panels b, $c$, and d. In the long axis view (Panel b), the aortic cusps are shown in the closed position in the center of the aortic lumen. In the short axis view (Panel c) only two cusps, anterior and posterior are seen; in diastole, a horizontal line of closure is seen, which did not alter with the change in the angulation of the transducer. $\mathrm{AOV}=$ aortic valve; $\mathrm{LA}=$ left atrium. 
root at the level of the aortic valve, the normal pattern of diastolic closure of the aortic valve was absent; only two aortic valve cusps, anterior and posterior, were recognized (Fig. 3c). These two cusps closed in the center of the aortic lumen during diastole along the $\mathrm{X}$-axis; changing the angulation of the transducer in various positions did not alter this pattern. In addition, increased reflectance of echoes along the line of diastolic closure was observed in the short axis view; this finding is probably caused by the well known redundancy of the leaflet tissue which results from the folding and unfolding of the elongated cusp margins in diastole. ${ }^{2,10)-12)}$ The horizontal closure

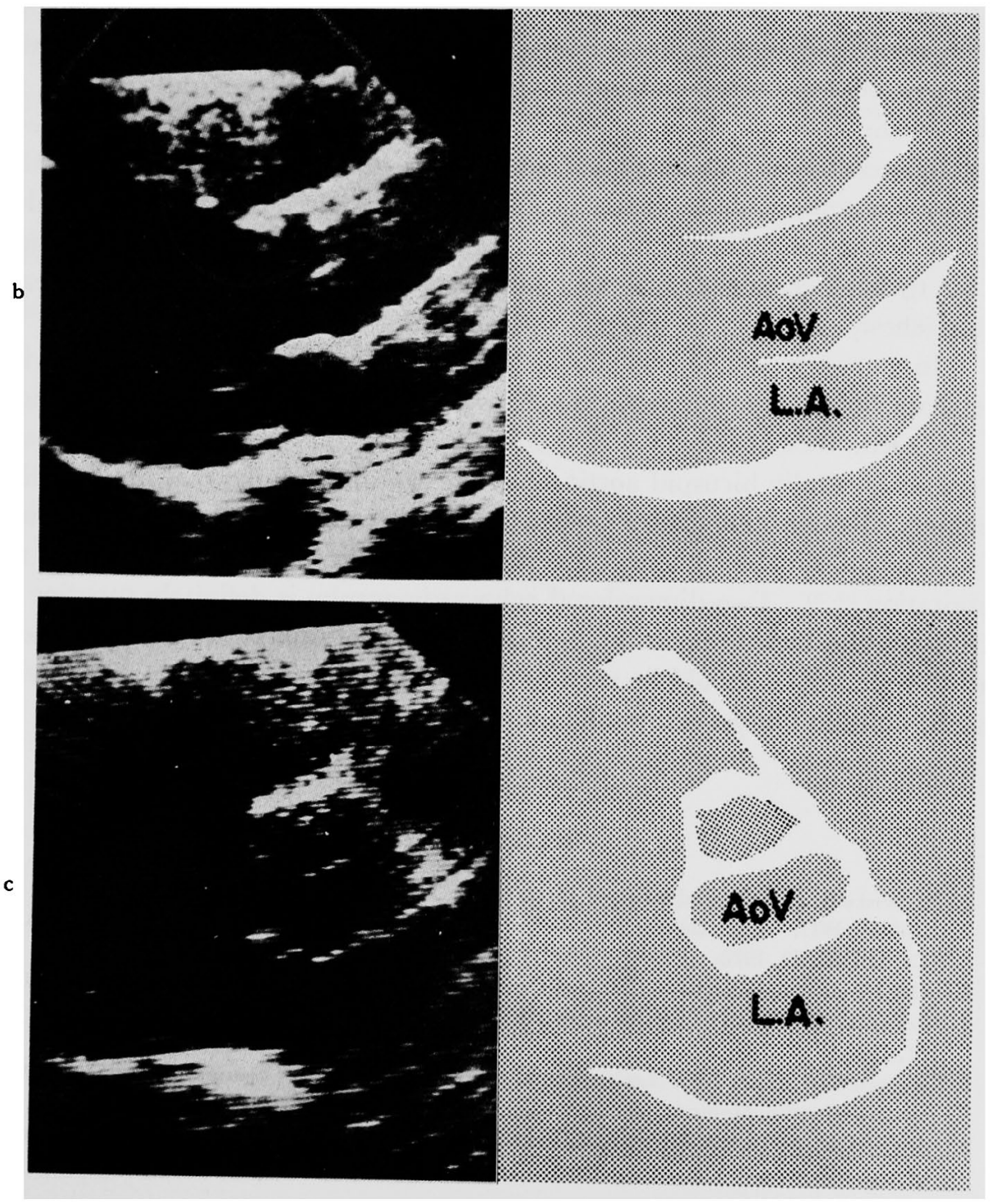


along the " $\mathrm{X}$ " axis was present in every patient; the normal tricuspid aortic valve, on the other hand, formed a " $\mathrm{Y}$ " pattern during diastole in all our normal controls. However, in order to adequately demonstrate these findings, diligent analysis of multiple still and real time frames of the short axis images of the aortic root at the level of the aortic valve is necessary.

The results of our study concur with those of Fowles et al, ${ }^{12)}$ who also demonstrated superiority of two-dimensional echocardiographic technique in the detection of bicuspid aortic valve. In their group of patients with angiographically proven stenotic and non-stenotic bicuspid aortic valves, the precise valve anatomy was identified by $\mathrm{M}$-mode echocardiography in $75 \%$ of subjects, and by two-dimensional echocardiography in $95 \%$ of subjects. ${ }^{12}$ ) In our series, two-dimensional echocardiography in the short axis view confirmed the diagnosis of a suspected congenitally bicuspid aortic valve in all patients, in spite of the normal M-mode echocardiogram in each patient. We believe that the criterion of an abnormal eccentricity index of M-mode echocardiography is insufficiently sensitive, probably because the position of aortic valve closure in the lumen of the aortic root varies with the position and angulation of the transducer on the chest wall with respect to the orientation of the heart in the chest, especially in subjects with chest deformities.

In summary, demonstration of only two cusps of the aortic valve, with the diastolic closure line along the $\mathrm{X}$ axis in the parasternal short axis view of the aortic root at the level of the aortic valve, appears to be the hallmark of the congenitally bicuspid aortic valve on two-dimensional real time cardiac imaging. Recognition of a bicuspid aortic valve condition is important in view of its susceptibility to subacute bacterial endocarditis, ${ }^{3), 4}$ ) aortic stenosis and regurgitation. ${ }^{51-9)}$ Two-dimensional echocardiography is definitely superior to M-mode echo technique in the detection and confirmation of this condition, and this technique should be used routinely for screening young patients with systolic ejection clicks and systolic murmurs.

\section{REFERENGES}

1. Koletsky S: Congenital bicuspid aortic valves. Arch Intern Med 67: 129, 1941

2. Roberts WC: The congenitally bicuspid aortic valve. A study of 85 autopsy cases. Am J Cardiol 26: 72, 1970

3. Osler $W$ : The bicuspid condition of the aortic valve. Trans Ass Am Physicians 2: 185, 1886

4. Lewis T, Grant RT: Observations relating to subacute infective endocarditis. Heart 10: 21,1923

5. Roberts $\mathrm{WC}$, Elliot LP: Lesions complicating the congenitally bicuspid aortic valve. Anatomic and radiographic features. Radiol Clin North Am 6: 409, 1968

6. Smith DE, Matthews MB: Aortic valvular stenosis with coarctation of the aorta. With special reference to the development of aortic stenosis upon congenital bicuspid valves. Brit 
Heart J 17: 198, 1955

7. Campbell M: Calcific aortic stenosis and congenital bicuspid aortic valves. Brit Heart J 30: 606, 1968

8. Schlant RC: Calcific aortic stenosis. Am J Cardiol 27: 581,1971

9. Edwards JE: The congenital bicuspid aortic valve. Circulation 23: 485, $196 \mathrm{I}$

10. Nanda NG, Gramiak R, Manning J, Mahoney EB, Lipchik EO, DeWeese JA: Echocardiographic recognition of the congenital bicuspid aortic valve. Circulation 49: 870, 1974

11. Radford DJ, Bloom KR, Izukawa T, Moes CAF, Rowe RD: Echocardiographic assessment of bicuspid aortic valves. Angiographic and pathological correlates. Circulation 53: 80, 1976

12. Fowles RE, Martin RP, Abrams JM, Schapira JN, French JW, Popp RL: Two-dimensional echocardiographic features of bicuspid aortic valve. Chest 75: 434, 1979

13. Gramiak R, Shah PM: Echocardiography of the normal and diseased aortic valve. Radiology $96: 1,1970$

14. Feigenbaum H: Aortic valve. in Echocardiography, Lea and Febiger, Philadelphia, p141164,1976

15. Weyman AE, Feigenbaum H, Dillon JC, Chang S: Cross-sectional echocardiography in assessing the severity of valvular aortic stenosis. Circulation 52: 828, 1975

16. DeMaria AN, Bommer W, Jaye J, Lee G, Bouteller J, Mason D: Value and limitations of cross-sectional echocardiography of the aortic valve in the diagnosis and quantification of valvular aortic stenosis. Circulation 62: 304, 1980

17. Leech G, Mills P, Leatham A: The diagnosis of a non-stenotic bicuspid aortic valve. Brit Heart J 40: 941, 1978

18. Tajik AJ, Seward J, Hagler D, Mier D, Lie JT: Two-dimensional real-time ultrasonic imaging of the heart and great vessels. Mayo Clin Proc 54: 271, 1978 